Abstract FRI0289 - Tabe 1

\begin{tabular}{|c|c|c|c|c|c|c|c|c|c|c|}
\hline & Age & OAC & APT & Vascular risk factors & Valvular disease & Brain stem & Frontal lobe & Strokes $(\geq 2)$ & Mortality & Follow-up \\
\hline CVD with APS & $57,48 \pm 21,52$ & $48 \%$ & $20 \%$ & $80 \%$ & $20 \%$ & $0 \%$ & $12 \%$ & $44 \%$ & $40 \%$ & $8,64 \pm 6,72$ \\
\hline CVD without APS & $\begin{array}{c}61,16 \pm 20,6 \\
\text { p } 0,76\end{array}$ & $\begin{array}{c}0 \% \\
p<001\end{array}$ & $\begin{array}{c}72 \% \\
p<001\end{array}$ & $\begin{array}{c}68 \% \\
\text { p } 0,33\end{array}$ & $\begin{array}{c}0 \% \\
\text { p } 0,02\end{array}$ & $\begin{array}{l}20 \% \\
\text { p0,02 }\end{array}$ & $\begin{array}{c}36 \% \\
\text { p } 0,04\end{array}$ & $\begin{array}{c}16 \% \\
\text { p } 0.03\end{array}$ & $\begin{array}{c}44 \% \\
\text { p } 0,77\end{array}$ & $\begin{array}{c}2,04 \pm 2,99 \\
p<001\end{array}$ \\
\hline
\end{tabular}

Methods: Retrospective and, descriptive study of patients with APS (Sidney criteria) and CVD followed for a long period of time in a specific Systemic Autoimmune Diseases and Thrombosis Unit. Subsequently, retrospective casecontrol study was performed. Case definition: patients with CVD attributable to APS. Control definition: patients with CVD without APS. The controls were matched with cases by sex and age (within the same decade). Chi-square and t-student were used, using the statistical package SPSS22.0.

Results: 25 patients (25/88 28,4\%) had CVD, 19 (76\%) of primary APS and $6(21 \%)$ of secondary APS. 17 patients $(71,6 \%)$ were female. The mean age was $57,48 \pm 21,52$ [range $13-89$ ], with a mean follow-up of $8,64 \pm 6,72$ years. $24 \%$ of patients had atrial fibrillation, $80 \%$ had one cardiovascular risk factor and $48 \%$ had two or more factors (hypertension 68\%, hypercholesterolemia 36\%, diabetes $20 \%$, tabaquism $4 \%$ ). Echocardiographic study was performed in $72 \%$ of patients with APS. Mitral valve was mainly involved. Most CVD were ischemic events (92\%). The brain areas most involved were the basal ganglia (36\%), together with the parietal and temporal lobe (16\% respectively). $40 \%$ had two or more affected regions. $44 \%$ of the patients had two or more episodes of stroke. Lupus anticoagulant was positive in $40 \%$, anticardiolipin antibodies in $76 \%$ and anti- $\beta 2$ glycoproteinl antibodies in $20 \%$. No differences were found with isotypes of APA and recurrent thrombosis or mortality. The treatment applied was oral anticoagulants (OAC) $(48 \%)$, antiplatelet therapy (APT) $(20 \%)$ and low molecular weight heparin (20\%). In 10 patients (40\%) CVD was diagnosed before APS (mean $8.64 \pm 6.7$ years). The mortality was $44 \%$ and $40 \%$ of the patients were hospitalized more than once. When we compared the groups: treatment, performed echocardiogram, valvular disease, affected brain areas, recurrent strokes and follow-up time, revealed significant differences (see table 1).OAC were more used in the patients with APS and APT was the most common in control group. Valvular disease was more frequent in case group. The brainstem and the frontal lobe were the areas more affected in patients without APS. The number of strokes was higher in APS group. The patients with CVD and APS had a long-term follow-up.

Conclusions: The prevalence of CVD in our series of APS was $28.4 \%$ and most often were ischemic events. Most of the patients were women with high recurrent strokes and mortality. No differences were found with isotypes of APA and recurrent thrombosis or prognosis. CVD with APS patients had more recurrent strokes and longer follow-up.

Disclosure of Interest: None declared

DOI: 10.1136/annrheumdis-2017-eular.4509

\section{FRI0290 INTERLEUKIN 10 GENE POLYMORPHISMS IN PRIMARY SJÖGREN SYNDROME IN A TUNISIAN POPULATION}

M. Tougorti ${ }^{1}$, A. Kallel ${ }^{2}$, I. Ben Ghorbel ${ }^{1}$, R. Jemaa ${ }^{2}$, M.H. Houman ${ }^{1}$, N. Kaabachi ${ }^{2}$. ${ }^{1}$ Internal Medicine; ${ }^{2}$ Biochemistry, Rabta university hospital, Tunis, Tunisia

Background: Primary Sjögren syndrome (PSS) is one of the most common autoimmune systemic rheumatic diseases although its prevalence anging between 0.6 and $1.7 \%$. PSS affects exocrine glands and lead to sicca syndrome. Interleukin-10 (IL-10) is a pleiotropic cytokine that is involved in the inflammation process of PSS.

Objectives: The aim of our study was to determine in a Tunisian population, clinical and biological characteristics of patients with primary PSS, allelic and genotypic frequencies of $(-1082 \mathrm{G} / \mathrm{A},-819 \mathrm{C} / \mathrm{T}$ and $-592 \mathrm{C} / \mathrm{A})$ polymorphisms in IL-10 gene and to evaluate the association of these polymorphism with PSS.

Methods: The population we studied consisted of 242 subjects with female predominance (average age at diagnosis $=49$ years), divided into 84 PSS patients (fulfilling the revised AECG criteria 2002 and/or ACR proposed criteria 2012), recruited in the internal medicine department of the Rabta hospital and 158 controls recruited in the Greater Tunis. II 10 level was assessed by ELISA. Polymorphisms genotyping of the IL-10 gene was done using PCR-RFLP technique.

Results: I110 plasma level was lower in PSS patients $(23.71 \mathrm{pg} / \mathrm{ml}, \mathrm{n}=73$ ) compared to healthy volunteers $(42.27 \mathrm{pg} / \mathrm{ml}, \mathrm{n}=60)$ and the difference was statistically significant $(p=0.01)$.

The genotype frequencies of our population respected Hardy-Weinberg equilibrium distribution both in patients by primary SS than in controls. In PSS patients, the genotype frequencies of $-592 \mathrm{C} / \mathrm{A}$ are $53 \%$ for the CC genotype, $41 \%$ for the $\mathrm{CA}$ and $6 \%$ for the AA genotype. In controls these frequencies are respectively $60.3 \%, 32.9 \%$ and $6.8 \%$. The genotype frequencies of $-1082 \mathrm{G} / \mathrm{A}$ are $29.6 \%$ for the AA genotype, $63 \%$ for the $A G$ and $7.4 \%$ for the GG genotype. In controls these frequencies are respectively $41.5 \%, 52.1 \%$ and $6.3 \%$.

The genotype frequencies of $-819 \mathrm{C} / \mathrm{T}$ are $47.6 \%$ for the CC genotype, $43.9 \%$ for the CT and $8.5 \%$ for the TT genotype. In controls these frequencies are respectively $41.5 \%, 52.1 \%$ and $6.3 \%$.

No significant differences in genotypic frequencies were observed between cases and controls in the three polymorphisms.
Statistical analysis preformed revealed that there was neither protective nor aggravating hapoltype. However ATC haplotype seems to have a protective impact in controls $(p=0.06$ and $O R=0.20)$

Conclusions: IL10 level was significantly higher in PSS patients in precedent studies (1) (2). In our case II10 level was associated with PSS in Tunisian patients but it was statistically lower than controls. Our results show that the three polymorphisms of gene of IL-10 are not a marker of SGS in the Tunisian population. This result might be explained by allelic variation or ethnic group. References:

[1] Marka M, Bessenyei B, Zeher M, Semsei I. IL-10 promoter -1082 polymorphism is associated with elevated IL-10 levels in control subjects but does not explain elevated plasma IL-10 observed in Sjogren's syndrome in a Hungarian cohort. Scandinavian journal of immunology. 2005;62(5):474-80.

[2] Vazquez-Villamar M, Palafox-Sanchez CA, Munoz-Valle JF, Valle Y, OrozcoBarocio G, Hernandez-Bello J, et al. Analysis of IL10 haplotypes in primary Sjogren's syndrome patients from Western Mexico: Relationship with mRNA expression, IL-10 soluble levels, and autoantibodies. Human immunology. 2015;76(7):473-9.

Disclosure of Interest: None declared

DOI: 10.1136/annrheumdis-2017-eular.6617

\section{FRI0291 CD4 AND CD8 COUNT IN PATIENTS WITH SYSTEMIC LUPUS ERYTHEMATOSUS - ASSOCIATIONS WITH INFECTION AND DISEASE ACTIVITY}

H. Baharuddin $^{1}$, N. Zainudin ${ }^{2}$, A. Ahmad ${ }^{2}$, A. Rosman ${ }^{2}$, M. Mohd Zain ${ }^{2}$.

${ }^{1}$ Rheumatology, Universiti Teknologi MARA; ${ }^{2}$ Rheumatology, Hospital Selayang, Selangor, Malaysia

Background: Systemic lupus erythematosus (SLE) is an autoimmune disease driven by the activation of autoreactive T and B cells. Decreased levels of CD4 counts were found in these patients, although there are conflicting data on whether CD4 is associated with SLE disease activity. ${ }^{1,2,3}$

Objectives: To investigate the associations between CD4 and CD8 counts with (1) active SLE and (2) infection in patients with SLE.

Methods: This was a cross-sectional study conducted in a rheumatology referral centre in Malaysia. Inclusion criteria included patients who fulfilled SLICC SLE classification and was admitted to rheumatology ward for active SLE or infection. We excluded patients who had overlap syndrome, retroviral disease or underlying malignancy. SLE Disease Activity Index (SLEDAI) score was assessed by the same rheumatologist.

Statistical analysis was performed using SPSS 20 and a p-value of $<0.05$ was considered significant. Pearson correlation was used to analyse associations between 2 continuous variables, while comparison between 2 continuous data was performed using student's t test.

Results: Forty-two patients participated in this study. Majority $(90.5 \%)$ were females with a mean age of $33.05( \pm 11.42)$ years and SLE duration of 8.69 $( \pm 5.26)$ years. There were $40(95.2 \%)$ ANA positive, $23(54.8 \%)$ anti-dsDNA positive, $13(31.0 \%)$ anti-smith positive, 24 (57.1\%) low C3 and $16(38.1 \%)$ low C4 in this cohort.

There were significant correlations between SLEDAI score and CD4 ( $r=-0.59$, $p<0.01)$ and CD8 counts $(r=-0.57, p<0.01)$. CD4 and CD8 counts were significantly lower in patients with clinically active SLE vs non-active, however, there were no significant differences in CD4 and CD8 counts in patients with infection vs no infection. We also found significantly high anti-dsDNA level and low complement 3 , but not complement 4 , in active SLE. White cell count was significantly higher in patients with infection. The details are tabulated in Table 1.

Table 1. CD4, CD8 and other parameters in infection and active SLE

\begin{tabular}{|c|c|c|c|c|c|c|}
\hline \multirow[t]{2}{*}{ Parameters } & \multicolumn{3}{|c|}{ Infection } & \multicolumn{3}{|c|}{ Active SLE } \\
\hline & Yes $( \pm S D), n=21$ & No $( \pm S D), n=21$ & $p$ & Yes $( \pm S D), n=26$ & No ( $\pm S D), n=16$ & $\mathrm{p}$ \\
\hline $\mathrm{CD} 4$ & $566.05(482.51)$ & $510.43(479.08)$ & 0.71 & $375.58(348.07)$ & $802.56(544.34)$ & $<0.01$ \\
\hline CD8 & 659.10 (562.91) & $456.48(221.86)$ & 0.13 & $406.0(346.04)$ & $804.44(460.04)$ & $<0.01$ \\
\hline SLEDAI & 7.14 (7.79) & $6.38(7.13)$ & 0.74 & $10.0(7.54)$ & $1.5(3.30)$ & $<0.01$ \\
\hline Anti-dsDNA & $110.17(145.84)$ & $103.14(186.87)$ & 0.91 & $149.01(193.48)$ & $42.47(53.62)$ & 0.05 \\
\hline C3 & $0.85(0.47)$ & 5) & 0.56 & $0.78(0.39)$ & 1.06 & 0.03 \\
\hline $\mathrm{C4}$ & 0 & 0 & 0.53 & 0.19 & 0.2 & 0.56 \\
\hline WBC & 8.92 (3.98) & $6.41(2.52)$ & 0.02 & 7.52 (3.72) & $7.90(3.30)$ & 0.74 \\
\hline
\end{tabular}

Conclusions: There were significant negative correlations between SLEDAI score and CD4 and CD8 counts, with low CD4 and CD8 counts found in patients with active SLE. There were no significant differences in CD4 and CD8 counts during infection.

\section{References:}

[1] Ferreira S, Vasconcelos J, Marinho A et al. CD4 lymphocytopenia in systemic lupus erythematosus. Acta Rheumatol Port 2009; 34: 200-206.

[2] Bonelli M, Savitskaya A, von Dalwigk K et al. Quantitative and qualitative 